\title{
Chronic elemental mercury intoxication: neuropsychological follow-up case study
}

\author{
M.-S. HUA, C.-C. HUANG ${ }^{\dagger}$ and \\ Y.-J. YANG
}

Department of Psychology, National Taiwan University, Taipei, Taiwan

$\dagger$ Department of Neurology, Chang Gung Memorial Hospital, Taipei, Taiwan

$\$$ Department of Neurology, Taipei Municipal Chung Shiao Hospital, Taipei, Taiwan

(Received 25 May 1995; accepted 1 August 1995)

In initial and follow-up investigations of neuropsychological function in a patient with elemental mercury intoxication, his scores were compared with those of a group of normal control subjects matched for sex, age and education. Each subject received a comprehensive neuropsychological examination including a personality inventory. On the initial examination the results indicated that the patient had a significant depression of performance intellectual functioning, impairments of attention, non-verbal short-term memory and visual judgement of angles and directions, psychomotor retardation and personality changes including depression, anxiety, desire to be alone, lack of interest and sensitivity to physical problems. Such an impairment picture is compatible with the previous observations of individuals with chronic exposure to elemental, organic or inorganic mercury. The follow-up study was undertaken about 1.5 years later. The results show that the patient's cognitive and personality functions were fully recovered. Our findings thus suggest a reversibility of impaired neuropsychological function in persons with elemental mercury poisoning if a prompt removal from the toxic environment is accomplished, together with proper medical treatment.

\section{Introduction}

Occupational exposure to mercury has been reported in miners, furcutters, mirror makers, gilders, hatters, dentists, and manufacturers of chlorine, chloralkali, thermometers, lamps, and thermonuclear weapons [1,2]. Neurotoxic effects of chronic exposure to mercury are well known. Generally, neuropathological changes in humans have been found in frontal and occipital cortices, basal ganglia, substantia nigra, superior temporal cortex, cerebellum, and subcortical white matter [3-6]. Grandjean [5] claimed that lesions resulting from elemental mercury intoxication were located in the occipital cortex and substantia nigra. Neurological deficits due to organic type poisoning include severe encephalopathy with persistent neurological disabilities [7] or a syndrome comparable to amyotrophic lateral sclerosis $[8,9]$, while inorganic or elemental mercury intoxication can produce polyneuropathy and tremor, and may further result in decreased visual acuity, particularly for night vision, slurred speech, disturbance of equilibrium, intention tremor, ataxic gait, and involuntary jerky movements if the disease progresses [10-12]. Neuropsychiatric problems due to inorganic mercury exposure include avoidant, irritable and overly sensitive interpersonal behaviour; depression; lassitude and

Correspondence to: Mau-Sun Hua, PhD, Department of Psychology, National Taiwan University, Taipei 10764, Taiwan. 
fatigue [13]. The term 'erethism' has been used to refer to such a constellation of emotional disturbances [2]. Neuropsychological impairments mainly involving defects of visual-motor coordination, abstract reasoning and memory, especially non-verbal short-term memory, and visuospatial abilities, have also been reported [13-15]. However, most of these cognitive and emotional problems were found in patients exposed to inorganic or organic, rather than elemental, mercury. Although there are a few studies [16,17] of persons exposed to elemental mercury, overall there is a paucity of information regarding the effects of such exposure on cognitive and emotional functions. Furthermore, there have been few longitudinal studies of neuropsychological consequences of chronic exposure to elemental mercury, and the reversibility of neurobehavioural changes after such chemical poisoning is as yet unclear, so in the present study we made an attempt to investigate these matters using a longitudinal approach.

\section{Materials and methods}

On 5 August 1991, a 29-year-old male with a 12th-grade education, who worked in a lampsocket manufacturing factory in northern Taiwan, complained of tremor and unsteady gait, for 4 months. He had suffered from severe gum pain, loss of body weight, general malaise and emotional instability for 2 years. Gingivitis, and prominent sweating on the palms and soles, were noted. Neurological examination showed a mild constriction of visual fields, slurred speech, prominent dysdiadochokinesia, dysmetria, dysynergia, postural and action tremor of both hands, and ataxic gait. The patient could not perform Tandem's walk. Laboratory tests revealed a normal haemogram, biochemistry, EEG, EKG, evoke potentials, chest radiography, brain CT and MRI. However, the mercury concentration was $237 \boldsymbol{\mu}_{\mathrm{g}} / 1$ in blood and $610 \boldsymbol{\mu}_{\mathrm{g} / 1}$ in $24-\mathrm{h}$ urine. The patient received D-penicillamine $150 \mathrm{mg}$ per day for 1 week and then $300 \mathrm{mg}$ per day for 7 weeks, and was followed in the outpatients department for 2 years. His gait problem improved, and disappeared 2 months later. The improvement of action tremor was slower and the condition took 4 months to abate. With D-penicillamine treatment, blood mercury concentrations showed a rapid decrease in the first 2 weeks, and then slowly returned to normal in the next 8 weeks. Monitoring of 24-h urinary mercury showed an increase in the first week and a slow decrease within 2 months. The clinical summary has been reported elsewhere [18]. The patient received a comprehensive neuropsychological examination at the Neuropsychology Laboratory, Department of Neurology, Chang Guang Memorial Hospital, Taipei on 11 August 1991, and a follow-up study on 5 March 1993 after cessation of exposure for about 1.5 years.

Thirty normal, healthy subjects, randomly selected volunteers and matched for sex, age and educational level in years with the patient, served as controls. Each subject also received a comprehensive neuropsychological examination at the Neuropsychology Laboratory, Department of Neurology, Chung Gung Memorial Hospital, Taipei on 11 August 1991. The tests, covering orientation, learning and memory, intelligence, language and communication, spatial perception, visual perception, visual attention, manual dexterity, information processing speed, and personality are as follows:

Orientation: Temporal Orientation (TO) [19] and Orientation to Personal Information and Place (OPIP) [20]; verbal learning and short-term memory: Serial Digit Learning (SRL) [19] and Word Sequence Learning (WSL) [21]; non- 
Table 1. Demographic characteristics of subject groups

\begin{tabular}{lcccccccc}
\hline & \multicolumn{3}{c}{ Age (years) } & & \multicolumn{3}{c}{ Education (years) } & \\
\cline { 2 - 4 } & Mean & SD & Range & & Mean & SD & Range & $n$ \\
\hline Patient & 29 & n.a. & n.a. & & 12 & n.a. & n.a. & 1 \\
Normals & 28.33 & $3 \cdot 48$ & $20-36$ & & $11 \cdot 50$ & $1 \cdot 96$ & $9-14$ & 30 \\
\hline
\end{tabular}

n.a. $=$ Not available.

verbal learning and short-term memory: Benton Visual Retention Test (BVRT) [22] and Continuous Recognition Memory (CRM) [23]; intellectual function: WAIS-R [24]; language and communication: Multilingual Aphasia Examination (MAE) [25] including Visual Naming (VN), Semantic Verbal Fluency (VF), Token Test (TT), Aural Comprehension (AC), Oral Reading (OR), Reading Comprehension (RC), Spontaneous Story Writing (SS), Spontaneous Writing of Name and Address (NA), Spontaneous Writing of Words and Phrases (SW), Writing to Dictation of Words (DW), Writing to Dictation of Sentences (DS), Writing from Copy of Words (CW) and Writing to Copy of Sentences (CS); spatial perception: Judgement of Line Orientation (JLO) [19] and Three-Dimensional Block Constructional-model (3-DBC) [19]; visual perception: Facial Recognition Test (FRT) [19] and Visual Form Discrimination (VFD) [19]; visual attention: Line Cancellation Test (LCT) [26], and Gordon Diagnostic System (GDS) [27], including a 6-min vigilance and distractibility subtest; manual dexterity and psychomotor function: Purdue Pegboard (PP) [28] and Finger Tapping Test (FT) [29]; information processing speed: Paced Auditory Serial Addition Task-Revised (PASAT-R) [30]; frontal function: Wisconsin Card Sorting Test-Short Form (WCST-S) [31]; personality function: Ko's Mental Health Questionnaire (KMHQ) [32], which is a self-report (true and false) format with a total of 282 items including 11 scales. These scales are Paranoia (KPa), Hypochondriasis (KHs), Psychopathic deviate (KPd), SelfConfidence (KSc), Inferiority (KI), Anxiety/Psychasthenia (KAp), Compulsiveness (KC), Sexual Suppression (KSs), Aggression (KAg), Self-Awareness/Ego Strength (KEs), and Independence (KId). A high score on the scales of KPa, KHs, KPd, KI, $\mathrm{KAp}, \mathrm{KC}, \mathrm{KS}$, and $\mathrm{KAg}$ indicates psychopathology, and a high score on the scales of KSc, KEs and KId reflects normal personality functioning. With regard to the instructions and scorings of these tests, please refer to the references. Before receiving the neuropsychological examination each subject was given written informed consent. A total of $3 \mathrm{~h}$ was needed for each subject to complete the testing in one session.

\section{Results}

For most of the neuropsychological measures the patient's test score was considered abnormal when it was 1.5 standard deviation below the mean score of the normal control group, while for the temporal orientation and the error score of the BVRT, an impaired score was defined as a score 1.5 standard deviation above the mean error score of the nominal control group. An impaired score on the scales of $\mathrm{KPa}$, $\mathrm{KHs}, \mathrm{KPd}, \mathrm{KI}, \mathrm{KAp}, \mathrm{KC}$, KSs and KAg of KMHQ was defined as the test score 1.5 standard deviation above the mean score of the normal control group, while a detective score on scales of $\mathrm{KSc}$, KEs, and KId was 1.5 standard deviation below 
Table 2. Comparisons of test results of orientation, learning and memory, and intelligence between normals and patient

\begin{tabular}{|c|c|c|c|c|}
\hline & \multicolumn{2}{|c|}{ Normals $(n=30)$} & \multicolumn{2}{|c|}{ Patient $(n=1)$} \\
\hline & Mean & SD & 1991 & 1993 \\
\hline \multicolumn{5}{|l|}{ Orientation } \\
\hline TO & $0 \cdot 3$ & 0.53 & 0 & 0 \\
\hline OPIP & $11 \cdot 6$ & 0.62 & 12 & 12 \\
\hline \multicolumn{5}{|l|}{ Intellectual functioning } \\
\hline \multicolumn{5}{|l|}{ WAIS-R } \\
\hline VIQ & $104 \cdot 93$ & $14: 83$ & 105 & 109 \\
\hline PIQ & $95 \cdot 17$ & $12 \cdot 52$ & $73 \dagger$ & 93 \\
\hline FSIQ & $101 \cdot 03$ & $14 \cdot 00$ & 91 & 103 \\
\hline \multicolumn{5}{|c|}{ Verbal learning and memory } \\
\hline SDL-12 & $13 \cdot 60$ & $7 \cdot 52$ & 14 & 19 \\
\hline \multicolumn{5}{|l|}{ WSL } \\
\hline Short-term memory & $53 \cdot 27$ & $5 \cdot 69$ & 46 & 59 \\
\hline 10-minute recall & $4 \cdot 73$ & $1 \cdot 28$ & 6 & 6 \\
\hline \multicolumn{5}{|c|}{ Non-verbal learning and memory } \\
\hline \multicolumn{5}{|l|}{ BVRT } \\
\hline Correct & $8 \cdot 17$ & $1 \cdot 05$ & $3 \dagger$ & 9 \\
\hline Error & $2 \cdot 27$ & $1 \cdot 44$ & $14 \dagger$ & 1 \\
\hline \multicolumn{5}{|l|}{ CRM } \\
\hline Hit & $37 \cdot 03$ & $2 \cdot 31$ & 34 & 36 \\
\hline False alarm & $12 \cdot 57$ & $11 \cdot 06$ & 11 & 13 \\
\hline Total correct & $86 \cdot 70$ & 6.98 & 83 & 83 \\
\hline
\end{tabular}

$\dagger$ Defective performance.

the mean score of the normal control group. Tables $2-5$ indicate the results. On the first neuropsychological examination the defective test scores include the PIQ of the WAIS-R, both correct and error scores of BVRT, JLO, both of correct and time scores of 3-DBC, the time score of LCT, both of omission and correct scores of vigilance and distractibility, Purdue Pegboard, Finger Tapping Tests, the first subtest $(2 * 8 \mathrm{~s})$ and total correct scores of PASAT-R. With regard to personality functioning, the elevated scores were evident on the KMHQ's scales of KHs, KPd, KI, KAp and KAg. Normal cognitive and personality functions, however, were shown in the follow-up study.

\section{Discussion}

On the first examination our patient manifested cognitive impairments including performance intellectual functioning, non-verbal short-term memory, visuospatial perception, visually sustained and selective attention, manual dexterity, information processing and psychomotor speed. These cognitive changes, especially for deficits of manual dexterity and psychomotor speed, however, might be partially due to the patient's tremor problem. Meanwhile, the patient showed some personality changes with depression, anxiety, desire to be alone, lack of interest, and hypochondriacal tendency. Since the patient was very cooperative during the time period of receiving neuropsychological examination, such personality changes seemed less likely to be secondary to his illness symptoms, such as general malaise. The non-verbal short- 
Table 3. Comparisons of test results of language, spatial perception, and visual perception between normals and patient

\begin{tabular}{|c|c|c|c|c|}
\hline & \multicolumn{2}{|c|}{ Normals $(n=30)$} & \multicolumn{2}{|c|}{ Patient $(n=1)$} \\
\hline & Mean & SD & 1991 & 1993 \\
\hline \multicolumn{5}{|c|}{ Language and communication } \\
\hline \multicolumn{5}{|l|}{ MAE } \\
\hline $\mathrm{VN}$ & $54 \cdot 27$ & $3 \cdot 78$ & 56 & 60 \\
\hline SR & $12 \cdot 20$ & $1 \cdot 19$ & n.a. & 14 \\
\hline VF & $36 \cdot 60$ & 6.61 & 35 & 41 \\
\hline TT & $42: 83$ & $1 \cdot 23$ & 40 & 42 \\
\hline AC & $17 \cdot 73$ & $0 \cdot 52$ & 16 & 18 \\
\hline OR & $17 \cdot 63$ & 0.49 & 18 & 18 \\
\hline $\mathrm{RC}$ & $17 \cdot 57$ & $0 \cdot 50$ & 17 & 17 \\
\hline SS & $29 \cdot 17$ & $8 \cdot 48$ & 19 & 33 \\
\hline NA & $17 \cdot 27$ & 0.58 & 18 & 18 \\
\hline SW & $12 \cdot 07$ & $3 \cdot 43$ & $12 \cdot 5$ & 15 \\
\hline DW & $9 \cdot 80$ & $1 \cdot 81$ & $10 \cdot 5$ & 11 \\
\hline DS & $12 \cdot 47$ & 0.82 & 13 & 13 \\
\hline $\mathrm{CW}$ & $15 \cdot 70$ & 0.54 & n.a. & 16 \\
\hline CS & $10 \cdot 97$ & $0 \cdot 18$ & n.a. & 11 \\
\hline \multicolumn{5}{|c|}{ Spatial perception } \\
\hline $\mathrm{JLO}$ & $23 \cdot 43$ & $3 \cdot 74$ & $16 \dagger$ & 25 \\
\hline \multicolumn{5}{|l|}{ 3-DBC } \\
\hline Correct & $28 \cdot 80$ & 0.61 & 29 & 29 \\
\hline Time(s) & $86 \cdot 80$ & $25 \cdot 27$ & $139^{\prime \prime} \dagger$ & $70^{\prime \prime}$ \\
\hline \multicolumn{5}{|c|}{ Visual perception } \\
\hline FRT & $45 \cdot 87$ & $4 \cdot 74$ & 49 & 41 \\
\hline VFD & $29 \cdot 47$ & $2 \cdot 57$ & 30 & 32 \\
\hline
\end{tabular}

n.a. $=$ Not available.

$\dagger$ Defective performance.

term memory and psychomotor problems evident in our patient due to chronic elemental mercury poisoning are respectively consistent with the observations of Smith and colleagues [17], and Langolf and co-workers [16]. The non-verbal shortterm memory problem of our patient was mainly reflected by his impaired performance on the Benton Visual Retention Test, which can also be used to measure immediate visual attention span [33]. Whether such a non-verbal short-term memory problem in our patient was secondary to his visual attention dysfunction is not known. If this is the case, the inclusion of measures of visually sustained and selective attention in a comprehensive neuropsychological test battery for examining workers or patients chronically exposed to elemental mercury might be necessary. Overall, the cognitive impairment picture of our patient is similar to that of workers or patients resulting from inorganic mercury intoxication [13-15]. Also, the findings of personality changes in our patient derived from the objective personality inventory plus the indirect evidence of irritability at home reported by his spouse seemed to further support the previous observations [2,13]

About 1.5 years after discontinuing exposure and receiving D-penicillamine treatment, the patient was re-examined with the same tests. The results indicated that both cognitive and emotional functions were back to normal. In a review 
Table 4. Comparisons of test results of visual attention, manual dexterity, and information processing speed between normals and patient

\begin{tabular}{|c|c|c|c|c|}
\hline & \multicolumn{2}{|c|}{ Normals $(n=30)$} & \multicolumn{2}{|c|}{ Patient $(n=1)$} \\
\hline & Mean & SD & 1991 & 1993 \\
\hline \multicolumn{5}{|l|}{ Visual attention } \\
\hline \multicolumn{5}{|l|}{ LCT } \\
\hline Right visual field & $30 \cdot 00$ & 0.00 & 30 & 30 \\
\hline Left visual field & $29 \cdot 87$ & $0 \cdot 35$ & 30 & 30 \\
\hline Total correct & $89 \cdot 83$ & $0 \cdot 38$ & 90 & 90 \\
\hline Time (s) & $51 \cdot 27$ & $23: 87$ & $105^{\prime \prime} \dagger$ & $45^{\prime \prime}$ \\
\hline \multicolumn{5}{|l|}{ GDS } \\
\hline \multicolumn{5}{|l|}{ Vigilance (6 $\mathrm{min})$} \\
\hline Commission & $1 \cdot 27$ & $2 \cdot 60$ & 2 & 1 \\
\hline Omission & 0.80 & $1 \cdot 37$ & $5 \dagger$ & 1 \\
\hline Correct & $29 \cdot 20$ & $1 \cdot 37$ & $25 \dagger$ & 30 \\
\hline \multicolumn{5}{|l|}{ Distractibility (6 min) } \\
\hline Commission & $4 \cdot 70$ & $11 \cdot 83$ & 5 & 3 \\
\hline Omission & $4 \cdot 77$ & $5 \cdot 88$ & $14 \dagger$ & 1 \\
\hline Correct & $25 \cdot 30$ & $5 \cdot 27$ & $17 \dagger$ & 29 \\
\hline \multicolumn{5}{|l|}{ Manual dexterity } \\
\hline \multicolumn{5}{|l|}{ PP } \\
\hline Dominant hand & $16 \cdot 43$ & $1 \cdot 41$ & $6 \dagger$ & 15 \\
\hline Non-dominant hand & $15 \cdot 13$ & $1 \cdot 38$ & $5 \dagger$ & 13 \\
\hline Both hands & $13 \cdot 10$ & $1 \cdot 32$ & $5 \dagger$ & 12 \\
\hline \multicolumn{5}{|l|}{ FT } \\
\hline Dominant hand & $59 \cdot 00$ & $10 \cdot 44$ & $24 \dagger$ & 56 \\
\hline Non-dominant hand & $52 \cdot 20$ & $8 \cdot 68$ & $28 \dagger$ & 59 \\
\hline \multirow{2}{*}{\multicolumn{5}{|c|}{$\begin{array}{l}\text { Information processing speed } \\
\text { PASAT-R }\end{array}$}} \\
\hline & & & & \\
\hline ISI $(2 \cdot 8 \mathrm{~s})$ & $22 \cdot 90$ & $3 \cdot 16$ & $16 \dagger$ & 23 \\
\hline ISI $(2 \cdot 4 \mathrm{~s})$ & $22 \cdot 37$ & $3 \cdot 32$ & 18 & 23 \\
\hline ISI $(2 \cdot 0 \mathrm{~s})$ & $22 \cdot 90$ & $4 \cdot 40$ & 15 & 25 \\
\hline ISI $(1.6 \mathrm{~s})$ & $20 \cdot 20$ & $5 \cdot 12$ & 14 & 22 \\
\hline Total correct & $85 \cdot 87$ & $14 \cdot 76$ & $63 \dagger$ & 93 \\
\hline
\end{tabular}

†Defective performance.

article, Hänninen [13], based on a series of cross-sectional study findings of her research team and other investigators, has addressed the issue of reversibility of motor and cognitive impairments, and emotional disturbances in workers due to chronic elemental, inorganic or organic mercury exposure. She claimed that motor problems are more reversible than cognitive and emotional changes because motor symptoms are easily identified and more often lead to cessation of exposure, which is not the case for workers with cognitive and emotional changes. In her view, the chance for cognitive and emotional function recovery is not high. Our findings, however, did not seem to support Hänninen's notion. The difference might be due to: (1) our findings derived from a follow-up case, rather than a cross-sectional type of research design; and (2) our subject was a patient with elemental mercury intoxication having neurological problems, rather than the workers exposed to elemental, inorganic, or organic mercury who did not manifest neurological symptoms. Future investigation of this issue is needed. 
Table 5. Comparisons of test results of frontal functioning and personality functioning between normals and patient

\begin{tabular}{|c|c|c|c|c|}
\hline & \multicolumn{2}{|c|}{ Normals $(n=30)$} & \multicolumn{2}{|c|}{ Patient $(n=1)$} \\
\hline & Mean & SD & 1991 & 1993 \\
\hline \multicolumn{5}{|l|}{ Frontal functioning } \\
\hline \multicolumn{5}{|l|}{ WCST } \\
\hline No. of complete categories & $5 \cdot 53$ & 1.66 & 6 & 7 \\
\hline No. of perseverative errors & $2 \cdot 50$ & $2 \cdot 45$ & 3 & 1 \\
\hline No. of non-perseverative errors & $6 \cdot 73$ & $4 \cdot 25$ & 4 & 5 \\
\hline No. of unique errors & $1 \cdot 27$ & $2 \cdot 52$ & 1 & 0 \\
\hline \multicolumn{5}{|l|}{ Personality functioning } \\
\hline \multicolumn{5}{|l|}{ KMHO } \\
\hline $\mathrm{KPa}$ & $6 \cdot 33$ & $2 \cdot 88$ & 6 & 2 \\
\hline $\mathrm{KHs}$ & $6 \cdot 77$ & $3: 64$ & $14 \dagger$ & 7 \\
\hline $\mathrm{KPd}$ & $6: 33$ & $3 \cdot 85$ & $12 \dagger$ & 6 \\
\hline $\mathrm{KSc}$ & $8 \cdot 50$ & $2 \cdot 84$ & 8 & 6 \\
\hline KI & $6 \cdot 20$ & $3 \cdot 78$ & $12 \dagger$ & 4 \\
\hline KAp & $5 \cdot 17$ & $4 \cdot 33$ & $17 \dagger$ & 1 \\
\hline $\mathrm{KC}$ & $8 \cdot 77$ & $4 \cdot 20$ & 14 & 8 \\
\hline KSs & $10 \cdot 73$ & $2 \cdot 85$ & 7 & 12 \\
\hline $\mathrm{KAg}$ & $5 \cdot 97$ & $3 \cdot 94$ & $14 \dagger$ & 6 \\
\hline KEs & $17 \cdot 10$ & $8 \cdot 88$ & 16 & 16 \\
\hline KId & $15 \cdot 70$ & $3 \cdot 61$ & 16 & 17 \\
\hline
\end{tabular}

tDefective performance.

In summary, this is the first follow-up case study to explore the issue of the reversibility of cognitive and emotional impairments in a patient with chronic elemental mercury intoxication. The general impairment picture from the initial neuropsychological examination seems to corroborate earlier findings in patients suffering with elemental, inorganic or organic mercury intoxication. A follow-up study of our patient shows a full recovery of both cognitive and emotional functions, suggesting a better prognosis in chemical elemental mercury intoxication than organic mercury intoxication. We further emphasized that the early removal from the exposure and early treatment might prevent the sequelae of cognitive and emotional alternations in patients with chronic elemental mercury intoxication.

\section{References}

1. Albers, J. W., Kallenbach, L. R., Fine, L. J. et al.: Neurological abnormalities associated with remote occupational elemental mercury exposure. Annals of Neurology, 124: 651-659, 1988.

2. Hartman, D. E.: Neuropsychological Toxicology, vol. 7 (Pergamon Press, New York), pp. 96-101, 1987.

3. Feldman, R. T.: Neurological manifestations of mercury intoxication. Acta Neurologica Scandinavica (Suppl.), 66: 201-209, 1982.

4. TAkeuchi, T., Ето, N. and Eто, K.: Neuropathology of childhood cases of methylmercury poisoning (Minamata disease) with prolonged symptoms with particular references to the decortication syndrome. Neurotoxicology, 1: 1-20, 1979.

5. Grandjean, P.: Behavioral toxicity of heavy metals. In P. Zbinden, V. Cuomo, G. Racagni and B. Weiss (editors) Application of Behavioral Pharmacology in Toxicology, (Raven Press, New York), pp. 331-340, 1983.

6. White, R. F., Feldman, R. G., Moss, M. B. et al.: Magnetic resonance imaging (MRI), neurobehavioral testing and toxic encephalopathy: two cases. Environmental Research, 61: 117-123, 1993. 
7. Balir, F., Damluji, S. F., Amin-Zaki, L. et al.: Methylmercury poisoning in Iraq. Science, 181: 230-241, 1973.

8. Brown, I. A.: Chronic mercurialism: a cause of the clinical syndrome of amyotrophic lateral sclerosis. Archives of Neurology and Psychiatry, 72: 674-681, 1954.

9. KAntaRjian, A. D.: A syndrome resembling ALS following chronic mercurialism. Neurology, 11: 639-644, 1961.

10. KARL, R.: Clinical and neurochemical aspects of inorganic mercury lointoxication. In: P. Vinken and G. Bruyn (editors) Handbook of Clinical Neurology, vol. 36 (Elsevier North-Holland: New York), pp. 147-197, 1979.

11. Vroom, F. Q. and Greer, M.: Mercury vapour intoxication. Brain, 95: 305-318, 1972.

12. WeIss, B.: Behavioral toxicology and environmental health science: opportunity and challenge for psychology. American Psychology, 38: 1174-1187, 1983.

13. HÄnninen, H.: Behavioral effects of occupational exposure to mercury and lead. Acta Neurologica Scandinavica (Suppl.), 66: 167-175, 1982.

14. Angotzi G., Camerino, D., CArboncini, F. et al.: Neurobehavioral follow-up study of mercury exposure. In: R. Gilioli, M. G. Cassitto and V. Foa (editors) Neurobehavioral Methods in Occupational Health (Pergamon Press, New York), pp. 247-253, 1982.

15. Uzzell, B. P. and Oler, J.: Chronic low-level mercury exposure and neuropsychology functioning. Journal of Clinical and Experimental Neuropsychology, 8: 581-593, 1986.

16. Langolf, G. D., Chaffin, D. B., Henderson, E. et al.: Evaluation of workers exposed of elemental mercury using quantitative tests of tremor and neuromuscular functions. American Industrial Hygiene Association Journal, 39: 976-984, 1978.

17. Smith, P. J., Langolf, G. D. and Goldberg, J.: Effects of occupational exposure to elemental mercury on short term memory. British Journal of Industrial Medicine, 40: 413-419, 1983.

18. Yang, Y.-J., HuAng, C.-J., SHIH, T.-S. et al.: Chronic elemental mercury intoxication: clinical and field studies in lampsocketicine manufactures. Occupational and Environmental Medicine, 51: 267-270, 1994.

19. Benton, A. L., Hamsher deS.K., Varney, N. R. et al.: Contributions to Neuropsychological Assessment: a clinical manual (New York: Oxford University Press), 1983.

20. Hamsher, DeS.K.: Orientation to Personal Information and Place (Department of Neurology, University of Wisconsin Medical School, Milwaukee), 1983.

21. HAmsher, DeS.K. and Roberts, R. J.: Word Sequence Learning (Department of Neurology, University of Wisconsin Medical School, Milwaukee), 1983.

22. Benton, A. L.: The Revised Visual Retention Test ( Psychological Corporation, New York), 1974.

23. Hannay, H. J. and Levin, H. S.: Continuous Recognition Memory Test (Department of Psychology, Auburn University, Alabama), 1979.

24. Wechsler, D.: Wechsler Intelligence Scale-Revised: Manual (Psychological Corporation, New York), 1981.

25. Benton, A. L. and Hamsher, DeS. K.: Multilingual Aphasia Examination (Department of Neurology, University Hospital of Iowa, Iowa City), 1978.

26. Hamsher, DeS. K.: Line Cancellation Test (Department of Neurology, University of Wisconsin Medical School, Milwaukee), 1978.

27. Gordon, M.: The Gordon Diagnostic System (Gordon System, DeWitt, NY), 1986.

28. Purdue Pegboard Foundation: Examiner's Manual for the Purdue Pegboard (Science Research Associates, Chicago, IL), 1948

29. Matthews, C. G. and Klove, H.: Instruction Manual for the Adult Neuropsychology Test Battery, (University of Wisconsin Medical School, Madison, WI), 1964.

30. Levin, H. S.: Paced Auditory Serial Addition Task-Revised (Neuropsychology Laboratory, Neurosurgery Division, University of Texas Medical Branch at Galveston, Galveston, TX), 1980.

31. Nelson, H. E.: A modified card sorting test sensitive to frontal lobe disease. Cortex, 12: 313-324, 1976.

32. Ko, Y.-H.: Ko's Mental Health Questionnaire (Chinese Behavioral Science, Taipei, Taiwan ), 1981.

33. LezaK, M. D.: Neuropsychological Assessment, 2nd edn (Oxford University Press, New York), pp. 451, 1983. 